\title{
Estratégia\&Negócios
}

ISSN 1984-3372

http://www.portaldeperiodicos.unisul.br/index.php/EeN/

\section{INTERAÇÃO UNIVERSIDADE-EMPRESA: UMA ANÁLISE DE EMPRESAS DA INDÚSTRIA DE SOFTWARE NO RIO GRANDE DO SUL}

\section{UNIVERSITY INDUSTRY LINKAGES: EVIDENCES FROM SOFTWARE INDUSTRY IN THE SOUTH OF BRAZIL}

\section{Daniel Pedro Puffal}

Doutor em Administração, Professor e Pesquisador no Programa de Pós Graduação em Administração da UNISUL e na Universidade FEEVALE.

E-mail: daniel.puffal@unisul.br

\section{Vilmar Antonio Gonçalves Tondolo}

Professor pesquisador no Programa de Pós-Graduação em Administração da Universidade de Caxias do Sul e na Universidade FEEVALE.

E-mail: vtondolo@gmail.com

\section{Dusan Schreiber}

Doutor em Administração, Professor e Pesquisador na Universidade FEEVALE.

E-mail: dusan@feevale.br

\section{Vania Gisele Bessi}

Doutora em Administração, Professora e Pesquisadora na Universidade FEEVALE.

E-mail: vania@feevale.br

Recebido em 13/05/2012. Aprovado em 31/08/2012. Disponibilizado em 01/10/2012. Avaliado pelo Sistema double blind review

R. eletr. estrat. neg., Florianópolis, v.5, n.2, p. 200-228, mai./ago. 2012 http://portaldeperiodicos.unisul.br/index.php/EeN/index
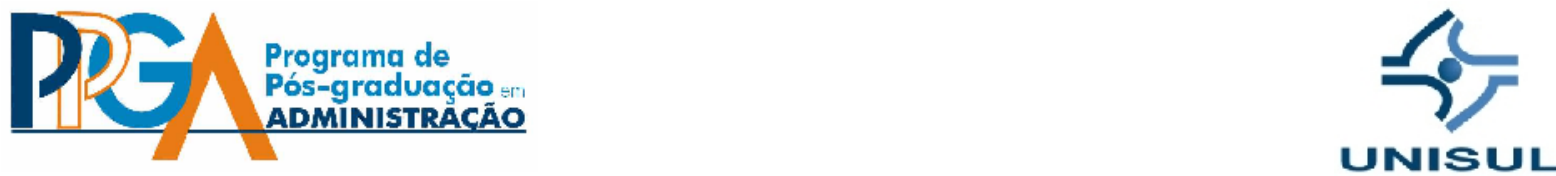

CCopyright 2008 UNISUL-PPGA/Estratégia e Negócios. Todos os direitos reservados. Permitida citação parcial, desde que identificada a fonte. Proibida a reprodução total. Em caso de dúvidas, consulte o editor: ademar.unisul@gmail.com; (48) 3229-193 
INTERAÇÃO UNIVERSIDADE-EMPRESA: UMA ANÁLISE DE EMPRESAS DA INDÚSTRIA DE SOFTWARE NO RIO GRANDE DO SUL

Daniel Pedro Puffal - Vilmar Antonio Gonçalves Tondolo - Dusan Schreiber - Vania Gisele Bessi

\section{RESUMO}

A indústria de software é caracterizada pela inovação, pelo uso intensivo de tecnologia e por sua proximidade das Instituições de Ensino Superior. Embora inserida em uma economia de mercado em que o principal locus da inovação seja a empresa, a história tem mostrado que, de um modo geral, elas não conseguem se capacitar sozinhas, sendo necessária a existência de um sistema nacional de inovação que as auxilie a desenvolver essa atividade. Nesse âmbito, a interação de empresas com universidades exerce um papel relevante. Por meio da análise de uma base de dados sobre interação universidade-empresa no Brasil e de um estudo de casos múltiplos, o artigo tem como objetivo observar a interação entre a universidade e as pequenas e médias empresas de software, atuantes no mercado internacional, localizadas no Rio Grande do Sul. Os resultados do estudo indicam que as interações das universidades com as Pequenas e Médias Empresas (PMEs) de software gaúchas atuantes no mercado internacional são menos intensas, mais informais e de menor complexidade, comparando com as interações desenvolvidas pelas demais empresas brasileiras. Observou-se ainda que há espaço para um avanço nas relações entre as empresas e as universidades no sentido do aumento do nível de inovação de seus produtos e processos. $\mathrm{O}$ artigo avança na compreensão do fenômeno estudado, revelando que, para os agentes pesquisados, apesar da proximidade física não foi observado o desenvolvimento de interações mais intensas.

Palavras-chave: Inovação. Interação universidade-empresa. Indústria de software.

\section{INTRODUÇÃO}

Alterações no ambiente em que as empresas se desenvolvem tem se intensificado, devido ao avanço da tecnologia de comunicação, permitindo acesso à informação e aumentando as possibilidades de interação com culturas distantes, gerando 
mudança no comportamento das empresas em relação aos mercados de atuação. Se por um lado estas mudanças proporcionam oportunidades de negócios sem precedentes para as empresas brasileiras, por outro, expõem o mercado doméstico para que empresas estrangeiras concorram em igualdade de condições com as empresas locais. Inúmeros trabalhos indicam que a competição global está deixando de ser uma opção e progressivamente está se transformando em uma exigência para empresas de diversos setores, obrigando-as a transpor fronteiras (PROCHNICK; ESVEVES; FREITAS, 2006). Apesar da internacionalização tardia das empresas brasileiras, a inserção no mercado internacional tem crescido aceleradamente em diversos setores da economia (FLEURY; FLEURY, 2007; PROCHNICK; ESVEVES; FREITAS, 2006). Logo, as empresas brasileiras da indústria de software não são exceção, pois enfrentam os concorrentes globais tanto no mercado interno quanto no externo.

A atuação das empresas de software no mercado internacional pode ser um dos caminhos para a adaptação à situação apresentada, uma vez que esta experiência, além de aumentar o mercado de atuação, pode também melhor preparar as empresas para desempenhar suas atividades no mercado doméstico. Para que a internacionalização ocorra, as empresas necessitam preparar-se tanto administrativamente quanto no que tange à produção, sendo que a inovação desempenha papel de fundamental importância. Sabe-se também das dificuldades administrativas que as empresas, especialmente as de menor porte, enfrentam quando expostas ao mercado internacional (PENROSE, 1959; MILLS et al., 2002).

Neste contexto, a inovação tem sido apresentada na literatura como uma fonte de sobrevivência e de expansão das empresas no mercado. A partir da contribuição seminal de Schumpeter (1949), em que à inovação é dado o principal papel na explicação do desenvolvimento econômico, novos avanços teóricos têm sido feitos para compreender melhor esse fenômeno.

A geração de inovação é facilitada na presença de infraestrutura tecnológica, na existência de recursos humanos qualificados, de relação de cooperação entre empresas e destas com outras instituições, de sistema de financiamento e de marco regulatório 
apropriado. O Sistema Nacional de Inovação - SNI constitui-se no arranjo institucional adequado a esse fim (LUNDVALL, 2007).

O conceito de Sistema Nacional de Inovação, discutido por economistas, tais como Freeman (1987), Lundvall (1994, 2007), Nelson (2006), entre outros, vem alcançando respeitabilidade no meio acadêmico e entre as instituições internacionais, sendo considerado um elemento importante na determinação da riqueza das nações, além de elemento para redução do custo de pesquisa e desenvolvimento nas empresas.

A interação entre a produção científica e a produção tecnológica desempenha um papel importante nos sistemas nacionais de inovação (MAZZOLENI; NELSON, 2007). Nos países desenvolvidos é possível identificar a existência de circuitos de retroalimentação positiva entre essas duas dimensões, tendo fluxos de informação e de conhecimento nos dois sentidos (COHEN; NELSON; WALSH, 2002). Universidades e institutos de pesquisa produzem conhecimento que é transmitido às empresas do setor produtivo, enquanto que a acumulação do conhecimento tecnológico produz questões importantes para a elaboração científica e na orientação da qualificação de recursos humanos. Já em países menos desenvolvidos, como o Brasil e outros, o sistema nacional de inovação ainda apresenta-se pouco dinâmico em termos de interações estabelecidas entre as universidades e as empresas (SUZIGAN; ALBUQUERQUE, 2011).

Tendo em vista o exposto, a partir de um estudo de casos múltiplos e análise de dados de um survey sobre a relação universidade-empresa no Brasil, o presente artigo tem como objetivo analisar a interação entre universidade e pequenas e médias empresas de software, atuantes no mercado internacional, instaladas no Rio Grande do Sul, e o papel desta interação no alcance dos benefícios ou resultados para essas empresas e as características que distinguem estas interações com as das demais empresas brasileiras.

Além do contexto de desenvolvimento tecnológico, em que a indústria de software desempenha papel importante, e da proximidade geográfica do setor em relação às universidades, justifica este trabalho a constatação de que, de acordo com o censo dos grupos de pesquisa de 2010, apenas $12,7 \%$ dos grupos de pesquisa registrados em 2010 no CNPq apresentavam alguma interação com empresas. Entretanto, a grande área de 
INTERAÇÃO UNIVERSIDADE-EMPRESA: UMA ANÁLISE DE EMPRESAS DA INDÚSTRIA DE SOFTWARE NO RIO GRANDE DO SUL

Daniel Pedro Puffal - Vilmar Antonio Gonçalves Tondolo - Dusan Schreiber - Vania Gisele Bessi

conhecimento denominada Engenharias apresentou um índice de interação com empresas de $30,1 \%$, mais que o dobro da média brasileira, dentro da qual as áreas de maior índice de interação são Engenharia Elétrica e Ciências da Computação (CNPQ, 2012).

Para tanto, além desta introdução, o trabalho se desenvolve em quatro seções: primeiramente discorre sobre a interação universidade-empresa, após são apresentados os métodos utilizados, em seguida um panorama da situação brasileira da interação universidade-empresa a partir dos resultados encontrados por uma pesquisa nacional com empresas com interação com grupos de pesquisa registrados no CNPq e os resultados da pesquisa empírica com empresas gaúchas de software. Finalmente, estão presentes as discussões e considerações finais do artigo bem como suas limitações e sugestões de trabalhos futuros.

\section{REFERÊNCIAL TEÓRICO}

\subsection{A INTERAÇÃO UNIVERSIDADE EMPRESA}

Universidades têm sido citadas como atores fundamentais nos sistemas de inovação (NELSON, 1993), e pesquisas realizadas nessas entidades vêm desempenhando um papel importante como fonte de conhecimento e no desenvolvimento de novas tecnologias aplicadas à indústria. Em reconhecimento a esse fato, governos de países industrializados realizam, desde 1970, várias iniciativas para aproximar as universidades às atividades de inovação industrial (MOWERY; SAMPAT, 2007). Parcela significativa dessas iniciativas visa a impulsionar o desenvolvimento econômico local baseado na pesquisa universitária, utilizando-se da criação de parques científicos, incubadoras, suporte a empresas incubadas, disponibilização de capital de risco e outras formas de instituições de apoio que proporcionam uma maior interação entre universidade e inovação industrial. 
INTERAÇÃO UNIVERSIDADE-EMPRESA: UMA ANÁLISE DE EMPRESAS DA INDÚSTRIA DE SOFTWARE NO RIO GRANDE DO SUL

Daniel Pedro Puffal - Vilmar Antonio Gonçalves Tondolo - Dusan Schreiber - Vania Gisele Bessi

Um dos papéis importantes das universidades, para o desenvolvimento de países, é o avanço na fronteira do conhecimento com vistas à aplicabilidade no setor produtivo. As universidades são fontes gerais de conhecimento necessários para as atividades de pesquisa básica (NELSON, 1990), bem como fontes de conhecimento especializado relacionado à tecnologia aplicadas nas empresas (KLEVORICK et al., 1995), além de serem responsáveis pela formação e treinamento de cientistas e engenheiros aptos a solucionar problemas relativos ao processo inovativo das empresas (ROSENBERG; NELSON, 1994). Outra contribuição importante das universidades, para o processo inovativo, é a geração de novas empresas de base tecnológica dentro do ambiente da universidade, denominadas spin-offs (STANKIEWICZ, 1994).

Klevorick et al. (1995) apresentam evidências empíricas sobre o papel das universidades e da ciência como uma importante fonte de oportunidades tecnológicas para a inovação industrial. Além disso, mostram como setores industriais distintos avaliam a importância relativa das universidades e da ciência para suas capacidades inovativas.

A interação universidade-empresa consolida e desenvolve o sistema nacional de inovação e deve ser compreendida como sua parte constituinte. Porém, a intensidade das relações depende da capacidade estrutural de absorção dos envolvidos, conforme Meyer-Kramer e Schmoch (1998). A característica da interação universidade-empresa é específica a cada país e depende da infraestrutura nacional de ciência e tecnologia. Para Rapini e Righi (2007), no Brasil, uma parte significativa dos relacionamentos na interação universidade-empresa tem um fluxo unidirecional, ou seja, é oriundo das universidades e instituições para as empresas.

\subsection{1 razões para a interação universidade empresa - IUE}

A decisão de participar de uma atividade de interação entre universidade e empresa - IUE não é simples, especialmente devido ao alto grau de incerteza envolvido no processo. De forma geral, os aspectos determinantes da IUE estão relacionados às 
características em nível da empresa e universidade, do setor e da localização dos agentes, também fortemente influenciado pelas políticas públicas pertinentes.

Um dos aspectos importantes para determinar a IUE é abordado pela Teoria Baseada em Recursos (BARNEY, 1991) que apresenta o entendimento de que os recursos internos da empresa desenvolvem papel importante no seu crescimento e prosperidade, bem como para a implantação da estratégia. Em outras palavras, as empresas necessitam desenvolver um conjunto de recursos e capacidades que suporte a implantação das suas estratégias, dentre elas, a de internacionalização. Caso não haja recursos internos na organização, esta irá buscá-los fora. Assim, a cooperação se dará apenas caso a empresa ou mesmo a universidade não consigam atingir seus objetivos de forma autônoma (AXELROD, 1984).

Observando a literatura existente, fortemente orientada para países desenvolvidos, percebe-se que os principais determinantes da IUE são as características da empresa (TETHER, 2002) e da universidade (MOWERY; SAMPAT, 2004), as características do setor (KLEVORICK et al., 1995; MALERBA, 2002 e 2004), a localização geográfica (ASHEIM; GERTLER, 2007; MYTELKA, 2000; TÖDTLING; TRIPPL, 2005), a área de conhecimento envolvida na interação (KLEVORICK et al., 1995; MEYER-KRAHMER; SCHMOCH, 1998) e políticas públicas (DODGSON, 1993).

Além dos determinantes citados, também influenciam na IUE as motivações e expectativas das organizações envolvidas. Esses determinantes podem ser distintos quando analisados pela ótica da empresa ou da universidade, assim, a seguir, são detalhados cada um dos determinantes para cada uma das organizações envolvidas na IUE.

A literatura indica que, para as empresas, um dos principais benefícios oriundos da cooperação entre universidade e empresa é a ampliação da capacidade em desenvolver tecnologia com menor investimento, em menor espaço de tempo e com menores riscos. Para Geisler (2001), uma expectativa presente nas empresas é a de efetuar o pagamento do investimento relativo à $\mathrm{P} \& \mathrm{D}$ (payoff) com resultados imediatos e não de longo prazo.

Bonaccorsi \& Piccaluga (1994) realizaram uma classificação das motivações para que empresas participem em relações interorganizacionais com a universidade. Os quatro 
principais aspectos desta classificação são: a) obter acesso às fronteiras científicas do conhecimento; b) aumentar o poder preditivo da ciência; c) Delegar, terceirizar ou dividir atividades específicas relacionadas ao desenvolvimento; e, d) Falta de recursos. Para Santoro (2000), o prestígio e o fortalecimento da imagem da empresa também são motivadores para a IUE.

A literatura também indica que, para as universidades, os benefícios mais expressivos oriundos da cooperação entre universidade e empresa são a maior possibilidade em captar recursos adicionais para a pesquisa básica e aplicada e a possibilidade de proporcionar um ensino vinculado aos avanços tecnológicos. Para Bonaccorsi e Piccaluga (1994), as expectativas mais importantes para as universidades são a geração, transição e propagação do conhecimento. Geisler (2001) complementa, afirmando que o que motiva as universidades à interação é a oportunidade de exposição dos alunos a problemas práticos reais; possibilidade de emprego para os graduandos; acesso à tecnologia, em que a indústria tem especial conhecimento; acesso ao financiamento de pesquisa, seja a empresa como fonte ou o governo, e acesso às capacidades industriais.

Com estes diversos determinantes e razões para que a interação universidadeempresa ocorra, é coerente que também seja diversa a forma de realização da interação.

\subsection{TIPOS DE INTERAÇÃO UNIVERSIDADE EMPRESA}

As pesquisas têm indicado que as relações de cooperação entre empresas e universidades variam com relação ao nível de pessoal envolvido, e recursos comprometidos (SANTORO, 2000), e essas relações englobam componentes como suporte à pesquisa, pesquisa cooperativa, transferência de conhecimento e transferência de tecnologia.

A partir da literatura existente na época, Bonaccorsi e Piccaluga (1994) constroem uma taxonomia dos tipos de relação universidade-empresa, cuja variável principal é a organização e preparação e comprometimento de recursos organizacionais em termos de pessoal, equipamento e recursos financeiros entre as duas partes envolvidas na relação. As autoras apresentam seis formas de cooperação classificadas de acordo com o recurso organizacional envolvido pela universidade, levando em conta os prazos de duração R. eletr. estrat. neg., Florianópolis, v.5, n.2, p. 200-228, mai./ago. 2012 
dos acordos e o grau de formalização: i) relações pessoais informais, sem o envolvimento da universidade; ii) relações pessoais formais, convênios entre universidade e empresa; iii) envolvimento de uma instituição de intermediação; iv) convênios formais com objetivo definido; v) convênios formais sem objetivo definido, tipo "guarda-chuva"; vi) criação de estruturas próprias para a interação.

Geisler (2001) ressalta que as interações entre universidade e empresa tornamse uma realidade organizacional quando os profissionais envolvidos transformam a interação em uma relação ou aliança cooperativa formal e bem estruturada, tornando-se parte da rotina nos processos de aquisição e integração de tecnologia. Assim, esta última passa a competir por recursos e atenção gerencial para o seu sucesso e sustentabilidade.

\subsection{O IMPACTO DA IUE NA EMPRESA E NA UNIVERSIDADE}

Os resultados obtidos da IUE podem ser distintos para cada um dos elementos da relação. De forma geral, empresas estão preocupadas com resultados de curto prazo enquanto que universidades tendem a tomar ações cujo resultado seja mais perceptível no longo prazo.

O resultado de uma IUE pode levar a uma inovação incremental em que o produto ou processo seja novo para a empresa, mas já existente no mercado ou também um incremento em alguma competência existente na empresa ou mesmo desenvolvimento de novas. Também pode advir de uma IUE uma inovação radical, na qual o resultado será novo não só para a empresa, mas também para o mercado como um todo (OECD, 2005). De acordo com o Manual de Oslo (2005), uma inovação tecnológica de produto é a implantação e comercialização de um produto com características de desempenho aprimoradas de modo a fornecer ao consumidor serviços novos ou aprimorados. Uma inovação de processo tecnológico é a implantação e adoção de métodos de produção ou de comercialização novos ou significativamente aprimorados (OECD, 2005). Por definição, toda inovação deve ter um grau de novidade, nomeados pelo Manual como produtos ou processos novos para a empresa, novos para o mercado e novos para o mundo. 
Estudos apresentam alguns resultados da IUE, tais como a troca temporária de posto de trabalho entre profissionais, a utilização de equipamentos e instalações das partes envolvidas, publicações em conjunto, seminários em conjunto, definições de padrões, ideias para novos projetos (GEISLER, 2001), patentes, licenciamento de produtos ou processos patenteados ou não (SANTORO, 2000), novos produtos, solução de problemas da empresa, invenções, inovações e spin-offs (BONACCORSI; PICCALUGA, 1994). Para Belderbos et al. (2004), a IUE também gera um aumento na receita da empresa advinda da venda de produtos novos, entretanto, seu impacto não é positivo na produtividade da empresa, sendo esta, se houver, decorrente de outros fatores não relativos à IUE.

Os resultados econômicos das pesquisas em universidades surgem de diferentes formas e setores, tais como informação tecnológica e científica, equipamentos e instrumentação, capacidades e capital humano, redes de capacidades científicas e tecnológicas, protótipos para novos produtos e processos, entre outros. Publicações de pesquisas, participação de conferências, geração de dissertações de mestrado e teses de doutorado e patentes são alguns dos resultados da IUE (EVANS et al., 1993; SANTORO, 2000). Além destas, Bonaccorsi e Piccaluga (1994) ainda acrescentam a geração de novos objetivos ou pesquisas a serem realizadas pela universidade.

Apesar das vantagens oriundas da interação das universidades com as empresas, existem algumas barreiras para que ela ocorra. Lundvall (2007) adverte para o perigo de a IUE ser encarada como uma fonte imediata de recursos para inovação. Isso pode restringir a autonomia acadêmica cuja função maior é a de educar e treinar alunos.

\section{PROCEDIMENTOS METOdOLÓGICOS}

Para o estudo da situação da IUE sob a ótica da empresa, realizou-se análise estatística descritiva do banco de dados de uma pesquisa nacional sobre IUE que teve por objetivo investigar a natureza da interação, sua sofisticação, diferenças entre as áreas da 
ciência e entre distintos setores industriais. As informações sobre empresas foram coletadas junto àquelas que mantiveram relação com as universidades declaradas no censo do CNPq de 2004. A coleta de dados foi realizada durante os anos de 2008 e 2009. Resultados preliminares da pesquisa foram publicadas por Fernandes et al. (2010).

Para o desenvolvimento da pesquisa relativa às empresas do setor de software, foram realizadas entrevistas com quatro empresas do setor. A escolha delas foi baseada no trabalho de pesquisa com micro e pequenas empresas (MPEs) de software localizadas no Rio Grande do Sul, estudadas em profundidade com relação ao modo de entrada no mercado internacional por Cortezia (2007), que, após uma pesquisa quantitativa, destacou oito MPEs com algum grau de internacionalização de acordo com a classificação de Coviello e Munro (1997). Estas oito empresas foram contatadas e apenas quatro aceitaram participar da pesquisa, número suficiente para um estudo de múltiplos casos com base na afirmação de Eisenhardt (1989) e Eisenhardt e Graebner (2007), os quais sugerem que apesar de não haver um número ideal de casos para análise, o interessante é que este número fique entre 4 e 10 casos.

Para a coleta de dados nas empresas, foi elaborado um roteiro para uma entrevista estruturada, com questões fechadas e abertas, buscando identificar a existência e a forma de interação das empresas com universidades ou instituições de ensino superior e institutos de pesquisa. As entrevistas foram realizadas por telefone, entre os meses de março a abril de 2009, sendo que o respondente eram os sócios proprietários da empresa.

As informações coletadas foram analisadas com a técnica de análise de conteúdo, segundo Bardin (1977). A análise de conteúdo é um conjunto de técnicas de análise de comunicações que visa, por meio de procedimentos sistemáticos e objetivos de descrição das mensagens, obter indicadores que permitam a inferência de conhecimentos relativos às condições de produção e recepção destas mensagens. A unidade de análise foi a interação da empresa com a universidade e quatro categorias de análises foram definidas, a saber: tipos de interação; razões para interação; resultados da interação e dificuldades para interação. 
INTERAÇÃO UNIVERSIDADE-EMPRESA: UMA ANÁLISE DE EMPRESAS DA INDÚSTRIA DE SOFTWARE NO RIO GRANDE DO SUL

Daniel Pedro Puffal - Vilmar Antonio Gonçalves Tondolo - Dusan Schreiber - Vania Gisele Bessi

\section{RESULTADOS}

\subsection{A INTERAÇÃO UNIVERSIDADE-EMPRESA NO BRASIL E AS EMPRESAS GAÚCHAS DE SOFTWARE}

Nesta seção, será apresentado um panorama geral da situação da interação universidade-empresa sob a ótica das empresas brasileiras e das empresas de software localizadas no Rio Grande do Sul. A percepção da IUE, sob a ótica de representantes das empresas, é apresentada a partir da base de dados da pesquisa nacional sobre IUE, em que 326 empresas brasileiras responderam à pesquisa. Já a situação da IUE na indústria de software foi obtida a partir da visão dos sócios proprietários de quatro MPEs de software localizadas no Rio Grande do Sul, que participaram do estudo de caso.

\subsubsection{A interação universidade-empresa na visão dos grupos de pesquisa brasileiros}

Para identificar o volume de cooperação entre universidade e empresas, foi realizado um levantamento no diretório dos grupos de pesquisa do CNPq. Foi possível observar que no censo realizado em 2010 pelo CNPq estavam registrados 27.523 grupos de pesquisa, dos quais apenas $12,7 \%$ declararam interação com empresas. As áreas com maior número de grupos são, respectivamente, Ciências Humanas, Ciências da Saúde, Engenharias e Ciências Sociais Aplicadas, como apresentado na Tabela 1

Tabela 1.

Tabela 1 - Grupos de pesquisa por grande área de conhecimento e índice de interação com empresas

\begin{tabular}{l|l|l|l|l|c}
\hline Grande área de conhecimento & $\begin{array}{l}\text { Número } \\
\text { grupos } \\
\text { (b) }\end{array}$ & $\begin{array}{l}\text { \% do total de } \\
\text { grupos } \\
\text { (c) }\end{array}$ & $\begin{array}{l}\text { Grupos integrados } \\
\text { com empresas } \\
\text { (d) }\end{array}$ & $\begin{array}{l}\text { Índice de } \\
\text { interação } \\
\text { (d/b) }\end{array}$ \\
\hline Ciências Humanas & $\underline{5.387}$ & $19,6 \%$ & $\underline{235}$ & $4,4 \%$ \\
Ciências da Saúde & $\underline{4.573}$ & $16,6 \%$ & $\underline{430}$ & $9,4 \%$ \\
$\underline{\text { Engenharias }}$ & $\underline{\underline{3.548}}$ & $12,9 \%$ & $\underline{1.068}$ & $30,1 \%$ \\
Ciências Sociais Aplicadas & $\underline{3.438}$ & $12,5 \%$ & $\underline{328}$ & $9,5 \%$ \\
(Continuação)
\end{tabular}


INTERAÇÃO UNIVERSIDADE-EMPRESA: UMA ANÁLISE DE EMPRESAS DA INDÚSTRIA DE SOFTWARE NO RIO GRANDE DO SUL

Daniel Pedro Puffal - Vilmar Antonio Gonçalves Tondolo - Dusan Schreiber - Vania Gisele Bessi

Tabela 1 - Grupos de pesquisa por grande área de conhecimento e índice de interação com empresas

\begin{tabular}{l|l|l|l|l|c}
\hline $\begin{array}{l}\text { Grande área de conhecimento } \\
\text { (a) }\end{array}$ & $\begin{array}{l}\text { Número } \\
\text { grupos } \\
\text { (b) }\end{array}$ & $\begin{array}{l}\text { \% do total de } \\
\text { grupos } \\
\text { (c) }\end{array}$ & $\begin{array}{l}\text { Grupos integrados } \\
\text { com empresas } \\
\text { (d) }\end{array}$ & $\begin{array}{l}\text { Índice de } \\
\text { interação } \\
\text { (d/b) }\end{array}$ \\
\hline Ciências Biológicas & $\underline{3.108}$ & $11,3 \%$ & $\underline{352}$ & $11,3 \%$ \\
Ciências Exatas e da Terra & $\underline{2.934}$ & $10,7 \%$ & $\underline{343}$ & $11,7 \%$ \\
Ciências Agrárias & $\underline{2.699}$ & $9,8 \%$ & $\underline{707}$ & $26,2 \%$ \\
\hline Linguística, Letras e Artes & $\underline{1.836}$ & $6,7 \%$ & $\underline{43}$ & $2,3 \%$ \\
\hline TOTAIS & $\mathbf{2 7 . 5 2 3}$ & $100 \%$ & $\mathbf{3 . 5 0 6}$ & $\mathbf{1 2 , 7 \%}$ \\
\hline
\end{tabular}

Fonte: Adaptado do Censo dos Grupos de Pesquisa do CNPq, 2010.

Entretanto, observa-se que o grau de interação dos grupos de pesquisa com as empresas tem uma elevada variação de acordo com a grande área de conhecimento classificada pelo CNPq. Observa-se na Tabela 1

Tabela 1 que a grande área de conhecimento que mais interage com as empresas é a área de Engenharias, com 30,1\% dos grupos com interação com empresas, seguida das Ciências Agrárias com 26,2\% dos grupos com interação. Já dentro da área de Engenharias, composta por 16 áreas de conhecimento, as que apresentaram maior índice de interação foram, respectivamente, Engenharia Elétrica, Ciência da Computação, Engenharia Civil, Engenharia de Materiais e Metalúrgica e Engenharia Mecânica.

O elevado índice de interação entre as universidades e empresas na grande área de conhecimento denominada Engenharias, reforça a importância da análise específica da área de software, uma vez que, a de Ciências da Computação pertence a esta grande área e apresenta um elevado índice de interação com a universidade. Isso pode ser observado no trabalho de Righi e Rapini (2011, p. 65), que apresentam dezoito "manchas de interação" na situação brasileira estando, entre elas a interação da área de Ciências da Computação com o setor de Serviços de Tecnologia da Informação.

Para compreender as razões, os tipos e os impactos da IUE nas empresas, a seguir são apresentados os resultados da análise da base de dados relativa à pesquisa realizada com os grupos de pesquisa registrados no CNPq, em que 1005 grupos de todo Brasil a responderam.

\subsection{A INTERAÇÃO UNIVERSIDADE-EMPRESA NA VISÃO DAS EMPRESAS BRASILEIRAS}


INTERAÇÃO UNIVERSIDADE-EMPRESA: UMA ANÁLISE DE EMPRESAS DA INDÚSTRIA DE SOFTWARE NO RIO GRANDE DO SUL

Daniel Pedro Puffal - Vilmar Antonio Gonçalves Tondolo - Dusan Schreiber - Vania Gisele Bessi

Quanto à localização, as 325 empresas que constituem a base de dados, concentram-se em maior número nas regiões Sudeste e Sul do Brasil, onde se localiza 79,7\% das empresas. Destas, 77 empresas pesquisadas estão localizadas no estado de Minas Gerais, 60 no Rio Grande do Sul, 60 em São Paulo, 29 em Santa Catarina, 18 no Paraná e 15 no Rio de Janeiro, ficando os restantes $18,3 \%$ distribuídos nos demais estados brasileiros. Quanto ao tamanho, de acordo com o critério para empresas industriais do SEBRAE - Serviço Brasileiro de Apoio as Micro e Pequenas Empresas, observa-se que há uma distribuição equilibrada, 34,2\% são grandes, $31,4 \%$ são médias e $33,5 \%$ são classificadas como pequenas e micro empresas. Quanto à origem do capital, 69,2 \%, é privado nacional, $12 \%$ privado estrangeiro, 5,8\% empresas públicas e o restante de capital misto.

As empresas da base de dados foram classificadas de acordo com ramo de atividade, composta majoritariamente por empresas da indústria de transformação $(62,8 \%)$, dividindo-se de maneira equilibrada entre as quatro categorias de intensidade tecnológica em que a OECD (2003) segmenta a atividade industrial.

Quanto à estrutura relativa à pesquisa e ao desenvolvimento das empresas $P \& D$, o número médio de empregados envolvidos nas atividades de $P \& D$ nas empresas da base de dados é de 28,5 empregados. Observou-se que $84,4 \%$ das empresas declararam que suas atividades de P\&D são contínuas e 67,1\% das empresas pesquisadas declararam possuir departamento de P\&D.

As empresas pesquisadas apresentam relações duradouras, pois 32,9\% das empresas têm cooperação com universidades ou institutos de pesquisa por um período entre 5 e 10 anos e 34,9\% das empresas afirmam ter relações com universidades ou institutos de pesquisa há mais de 10 anos.

\subsubsection{Razões da empresa para interação com universidades}


INTERAÇÃO UNIVERSIDADE-EMPRESA: UMA ANÁLISE DE EMPRESAS DA INDÚSTRIA DE SOFTWARE NO RIO GRANDE DO SUL

Daniel Pedro Puffal - Vilmar Antonio Gonçalves Tondolo - Dusan Schreiber - Vania Gisele Bessi

As razões que levam à colaboração da empresa com a universidade são diversas. Na Tabela 2,Tabela as razões estão listadas por ordem de importância, com a apresentação da média para as respostas das empresas brasileiras.

Tabela 2 - Razões da colaboração da empresa com universidades pesquisa

\begin{tabular}{l|l}
\hline Razões para colaboração com universidades & $\begin{array}{c}\text { Média } \\
\text { importância }\end{array}$ \\
\hline $\begin{array}{l}\text { Realizar testes necessários para produtos e processos da empresa. } \\
\text { Utilizar recursos disponíveis nas universidades e laboratórios de pesquisa. }\end{array}$ & 2,71 \\
Buscar conselhos de cunho tecnológico ou consultoria com pesquisadores e/ou professores para & 2,68 \\
solução de problemas relacionados à produção. & 2,65 \\
Contratar pesquisas que a empresa não pode realizar. & 2,62 \\
Transferência de tecnologia da universidade. & 2,61 \\
Contratar pesquisas para as atividades inovativas. & 2,56 \\
Aumentar a habilidade da empresa para encontrar e absorver informações tecnológicas. & \\
Conseguir informações sobre engenheiros ou cientistas e/ou tendências de P\&D nas áreas & 2,35 \\
científicas. & \\
Fazer, o mais cedo possível, contatos com estudantes universitários de excelência para futuro & 2,15 \\
recrutamento. &
\end{tabular}

Fonte: Adaptado da Base de dados da Pesquisa Nacional Interação Universidade-Empresa, 2010.

Observa-se que a razão mais importante é a realização de testes necessários para produtos e processos da empresa, com média de 2,71 pontos dos 4 possíveis, seguida da utilização de recursos disponíveis nas universidades e laboratórios de pesquisa, busca de conselhos de cunho tecnológico ou consultoria para solução de problemas relacionados à produção. Em quarta posição está a contratação de pesquisas que a empresa não pode realizar.

Dadas as respostas das empresas, observa-se que há uma maior preocupação na busca de recursos, conforme prevê o trabalho de Bonaccorsi e Piccaluga (1994), bem como na ampliação da capacidade de desenvolver tecnologia com menor investimento, menor prazo e menores riscos (GEISLER, 2001). Entretanto, contrariando a teoria, não se percebeu preocupação em aumentar o poder preditivo da ciência (BONACCORSI; PICCALUGA, 1994). 


\subsubsection{Tipos de interação com universidades}

As empresas assinalaram a importância dos tipos de interação com universidades, cujas médias são apresentas na Tabela 3

Tabela, por ordem de importância.

Tabela 3 - Interações e suas fontes de informação de universidades

\begin{tabular}{l|c}
\hline Tipo de interação com universidades & Importância \\
\hline Pesquisa realizada em conjunto com a universidade & 2,94 \\
Publicações e relatórios & 2,90 \\
Pessoal contratado com graduação ou pós-graduação & 2,73 \\
Conferências públicas e encontros & 2,67 \\
Troca informal de informações & 2,67 \\
Pesquisa encomendada à universidade & 2,52 \\
Consultoria com pesquisadores individuais & 2,45 \\
Participação em redes que envolvam universidades & 2,36 \\
Patentes & 2,10 \\
Parques científicos e/ou tecnológicos & 2,03 \\
Tecnologia licenciada & 2,02 \\
Intercâmbio temporário de pessoal & 1,95 \\
Incubadoras & 1,71 \\
Empresa pertencente a uma universidade & 1,52 \\
Empresa é spin-off da universidade & 1,48 \\
\hline
\end{tabular}

Fonte: Adaptado da Base de dados da Pesquisa Nacional Interação Universidade-Empresa, 2010.

Observa-se na Tabela 3 que pesquisa realizada em conjunto com a universidade é o tipo de interação mais importante para as empresas brasileiras, com média de 2,94 pontos dos 4 possíveis. Em seguida surgem as interações com uso de publicações e relatórios, contratação de pessoal da universidade, conferências públicas, troca informal de informações, pesquisa à universidade e consultoria com pesquisadores individuais.

A importância dos tipos de interação, para as empresas, é compatível com a classificação realizada por Bonaccorsi e Piccaluga (1994). Entretanto, chama atenção a baixa importância das relações com a universidade com uso de informações sobre patentes. Talvez esse resultado seja reflexo da fragilidade da estrutura brasileira de registro de propriedade.

Observa-se, também, que as interações que têm importante apoio em termos de estrutura colocada à disposição das empresas, tais como parques científicos ou tecnológicos, incubadores, empresas pertencentes à universidade, ou spin-off da universidade, 
INTERAÇÃO UNIVERSIDADE-EMPRESA: UMA ANÁLISE DE EMPRESAS DA INDÚSTRIA DE SOFTWARE NO RIO GRANDE DO SUL

Daniel Pedro Puffal - Vilmar Antonio Gonçalves Tondolo - Dusan Schreiber - Vania Gisele Bessi

apresentaram pouca importância para as empresas. Estas estruturas consomem importantes recursos públicos e aparentemente não tem a devida importância por parte das empresas.

\subsubsection{Impacto das interações universidade-empresa nas empresas}

Para perceber o desempenho tecnológico, as empresas foram questionadas quanto à introdução de produtos e processos novos ou aperfeiçoados nos últimos três anos. A Tabela 4

Tabela apresenta o desempenho relativo aos produtos, na qual se observa que 10,5\% das empresas não realizaram nenhum aperfeiçoamento ou desenvolvimento de produto novo, $63,1 \%$ realizaram aperfeiçoamento de produto existente, $47,7 \%$ introduziram produto novo para a empresa, $41,2 \%$ produto novo para o país e $19,1 \%$ produto novo para o mundo. Quanto as respostas pelas empresas ao questionário referente à questão do período em que ocorreram as introduções citadas, permitiu-se que fosse assinalada mais de uma alternativa, caso a empresa tivesse introduzido mais de um tipo de inovação nos últimos três anos.

Tabela 4 - Introdução de produtos novos ou aperfeiçoados nos últimos três anos

\begin{tabular}{l|r|l}
\hline Tipo de inovação em produto & Frequência & $\%$ \\
\hline Nenhum produto novo. & 34 & 10,5 \\
Aperfeiçoamento de um produto já existente. & 205 & 63,1 \\
Produto novo para a empresa, mas não para o país. & 155 & 47,7 \\
Produto novo para o país, mas não para o mundo. & 134 & 41,2 \\
Produto novo para o mundo. & 62 & 19,1 \\
\hline
\end{tabular}

Fonte: Adaptado da Base de dados da Pesquisa Nacional Interação Universidade-Empresa, 2010.

Com relação à inovação em processos, a Tabela 5Tabela 5 indica que, no Brasil, $8,6 \%$ das empresas não realizaram nenhum desenvolvimento de processo novo, $68,6 \%$ aperfeiçoou o processo existente, $43,1 \%$ desenvolveu processo novo para empresa, $24,3 \%$ processo novo para o país e $11,1 \%$ processo novo para o mundo.

Tabela 5 - Introdução de processos novos ou aperfeiçoados nos últimos três anos

\begin{tabular}{l|l|l}
\hline Tipo de inovação em processo & Frequência & $\%$ \\
\hline Nenhum processo novo. & 28 & 8,6 \\
Aperfeiçoamento de um processo já existente. & 223 & 68,6 \\
R. eletr. estrat. neg., Florianópolis, v.5, n.2, p. 200-228, mai./ago. 2012 & &
\end{tabular}

R. eletr. estrat. neg., Florianópolis, v.5, n.2, p. 200-228, mai./ago. 2012 
INTERAÇÃO UNIVERSIDADE-EMPRESA: UMA ANÁLISE DE EMPRESAS DA INDÚSTRIA DE SOFTWARE NO RIO GRANDE DO SUL

Daniel Pedro Puffal - Vilmar Antonio Gonçalves Tondolo - Dusan Schreiber - Vania Gisele Bessi

Processo novo para a empresa, mas não para o país.

Processo novo para o país, mas não para o mundo.

\begin{tabular}{|r|r}
140 & 43,1 \\
79 & 24,3 \\
36 & 11,1
\end{tabular}

Processo novo para o mundo.

24,3

11,1

Fonte: Adaptado da Base de dados da Pesquisa Nacional Interação Universidade-Empresa, 2010.

\subsection{A INTERAÇÃO UNIVERSIDADE-EMPRESA NA VISÃO DE MPES DE SOFTWARE DO RS}

Das 8 empresas indicadas no trabalho de Cortezia (2007), apenas 4 estavam dispostas a participar da pesquisa. Estas empresas são caracterizadas por serem MPEs, de acordo com os critérios do SEBRAE, devido a seu número de colaboradores e ao nível de faturamento. O Quadro 1 apresenta as descrições das empresas e permite observar que há uma diversificação geográfica, de tamanho, de idade das empresas, de experiência e de mercado-alvo.

Quadro 1 - Características das empresas pesquisadas

\begin{tabular}{|c|c|c|c|c|}
\hline Característica & Empresa 1 & Empresa 2 & Empresa 3 & Empresa 4 \\
\hline Cidade & Porto Alegre & São Leopoldo & Caxias do Sul & Novo Hamburgo \\
\hline Colaboradores & 45 & 17 & 50 & 40 \\
\hline $\begin{array}{l}\text { Faturamento } \\
\text { anual }\end{array}$ & $\begin{array}{l}\text { De } R \$ 434 \text { mil a } \\
R \$ 2,13 \text { milhões }\end{array}$ & $\begin{array}{l}\text { De } \mathrm{R} \$ 434 \text { mil a } \mathrm{R} \$ \\
2,13 \text { milhões }\end{array}$ & $\begin{array}{l}\text { De } \mathrm{RS} 2,13 \text { a } \mathrm{R} \$ \\
10,5 \text { milhões }\end{array}$ & $\begin{array}{l}\text { De } R \$ 434 \text { mil a } R \$ \\
2,13 \text { milhões }\end{array}$ \\
\hline Idade & 4 anos & 5 anos & 13 anos & 19 anos \\
\hline $\begin{array}{l}\text { Atividades } \\
\text { desenvolvidas }\end{array}$ & Teste de software & $\begin{array}{l}\text { Desenvolvimento } \\
\text { de software por } \\
\text { encomenda }\end{array}$ & $\begin{array}{l}\text { Desenvolvimento } \\
\text { de CAD para layout } \\
\text { de móveis }\end{array}$ & $\begin{array}{l}\text { Desenvolvimento e } \\
\text { implantação de } \\
\text { sistemas de ERP }\end{array}$ \\
\hline $\begin{array}{l}\text { Experiência } \\
\text { internacional }\end{array}$ & $\begin{array}{l}\text { Prestação } \\
\text { serviços }\end{array}$ & Joint-venture & $\begin{array}{ll}\text { Exportação } & \text { de } \\
\text { produto próprio }\end{array}$ & $\begin{array}{ll}\text { Exportação } & \text { de } \\
\text { produto próprio } & \end{array}$ \\
\hline $\begin{array}{l}\text { Mercados } \\
\text { externos que atua }\end{array}$ & Portugal & Itália & $\begin{array}{l}\text { Portugal, México e } \\
\text { Argentina }\end{array}$ & $\begin{array}{l}\text { México, China e } \\
\text { Europa }\end{array}$ \\
\hline
\end{tabular}

Fonte: Elaborado pelos autores com dados da pesquisa de campo, 2012.

As principais questões da entrevista versavam sobre o desenvolvimento de produtos e processos pela empresa, uso dos mecanismos de IUE, motivações da empresa para a interação com universidades e sobre os empecilhos ou fatores que impedem ou dificultam a interação.

\subsubsection{Razões para interação com universidades}


Verificou-se que a motivação das empresas de software pesquisadas para a interação com a universidade está associada às oportunidades emergentes. Razões ligadas principalmente à possibilidade de acesso a feiras de negócios, missões internacionais, utilização de espaços físicos em parques tecnológicos e incubadoras.

Das empresas pesquisadas, três indicaram que foram motivadas a aproximar-se da universidade para qualificar-se a ter acesso a alguma oferta de recurso público. Ou seja, a existência de edital público de subvenção, em que para a empresa participar se fazia necessária a interveniência de uma universidade. Percebe-se que a falta de recursos preconizada por Bonaccorsi e Piccaluga (1994) e a busca de resultados imediatos (GEISLER, 2001) são motivadores presentes nestas empresas.

Para os entrevistados, os aspectos de acesso às fronteiras do conhecimento na área de tecnologia da informação (BONACCORSI e PICCALUGA, 1994) não foram citadas, bem como também não foram à possibilidade de acesso aos novos processos gerenciais. As empresas entenderam que as relações com as universidades não trariam conhecimentos maiores do que aqueles que as pessoas das empresas já possuíam.

Questionadas quanto à influência da interação com a universidade no processo de internacionalização da empresa, uma vez que todas mantêm algum nível de internacionalização, nenhuma delas atribuiu importância à interação neste processo. As empresas entendem que a inserção internacional está associada às competências técnicas e organizacionais presentes nas empresas, sendo esses fatores facilitadores para o processo de internacionalização das empresas, além da existência na empresa de produtos inovadores e diferenciados como um aspecto fundamental no processo.

Dada a característica do produto gerado pelas empresas pesquisadas, intensivas em tecnologia, buscou-se identificar a forma de desenvolvimento deles. Nenhuma das empresas entrevistadas possui estrutura específica destinada ao desenvolvimento e à pesquisa, ocorrendo assim uma centralização desta atividade nos sócios das empresas que possuem formação específica na área de tecnologia da informação.

Todas as empresas entrevistadas afirmaram possuir alguma interação com instituições de ensino superior - IES, cujos mecanismos são indicados a seguir. 
INTERAÇÃO UNIVERSIDADE-EMPRESA: UMA ANÁLISE DE EMPRESAS DA INDÚSTRIA DE SOFTWARE NO RIO GRANDE DO SUL

Daniel Pedro Puffal - Vilmar Antonio Gonçalves Tondolo - Dusan Schreiber - Vania Gisele Bessi

\subsubsection{Tipos de interação com universidades}

Observou-se que os mecanismos de maior ocorrência nas empresas pesquisadas são as trocas informais de informações com a universidade, a participação em cursos oferecidos pelas IES e o apoio da empresa a projetos de pesquisa de estudantes de universidades. Os entrevistados ainda revelam que consultorias individuais realizadas por professores da universidade, bolsas de estudos para graduação ou pós-graduação sustentadas pela empresa e atividades com ex-alunos da IES em atividades na empresa são importantes. Os entrevistados revelaram que as interações variam em seu grau de intensidade.

Apenas uma das quatro empresas citou que contrato de consultoria ou de serviços de cunho tecnológico formais pela IES e contração formal de pesquisa realizada pela universidade com objeto definido é uma importante forma de interação com a universidade. Além disso, uma das empresas afirmou que a participação em polos, parques tecnológicos, incubadoras é importante, mas quando se aprofundou a discussão em relação à sua utilização de espaços em parques tecnológicos ou incubadoras, esta revela que seu maior interesse é o status que a localização proporciona à empresa.

Pode-se afirmar que as interações indicam que não há uma estruturação neste processo, pois são interações simples, de pouca intensidade, comprometimento ou complexidade. São interações mais informais (BONACCORSI; PICCALUGA, 1994), mais distantes da apresentada por Geisler (2001) em que a aliança cooperativa formal e bem estruturada torna-se parte da rotina dos processos de aquisição e integração da tecnologia, sustentável e de sucesso.

\subsubsection{Impacto das interações com universidades}


As empresas foram questionadas com relação ao resultado que a interação com a universidade gerou para a mesma. As empresas afirmaram que o principal resultado da interação com a universidade é a melhoria da imagem pública da empresa, o que coaduna com a afirmação de Santoro (2000). Em segundo lugar, o acesso aos laboratórios, às bibliotecas, aos instrumentos que a universidade dispõe, além de que o contato com o meio universitário estimula a criatividade na empresa.

Em terceiro lugar, as empresas informam que a interação com a universidade permite o acesso às fronteiras científicas do conhecimento, o atendimento a carências de recursos humanos e a algumas carências de recursos financeiros da empresa.

Resultados que as empresas entendem que são dificilmente alcançados foram: a divisão do risco com a universidade, a redução do custo da pesquisa em função de ter sido desenvolvida em conjunto com a universidade, bem como a redução do prazo necessário para desenvolver a tecnologia, além do acesso a pesquisas anteriores que obtiveram resultados satisfatórios. Estes resultados podem ser entendidos como os que mais justificariam a interação das empresas de tecnologia com as universidades (KLEVORICK et al., 1995).

Importa ressaltar que os aspectos em que os respondentes não concordaram com a afirmação de que podem ser motivos da integração: a redução dos prazos para desenvolvimento de tecnologia, o acesso aos resultados de pesquisas anteriores, a redução de custo da pesquisa e a divisão de risco com a universidade são importantes resultados da interação.

A empresa 2 informa que a relação com a universidade propiciou o acesso às rodadas internacionais de negócios, expondo a empresa a novos mercados. Além disto, a existência da interação da empresa com a universidade serviu como um "certificado de qualidade" transferindo credibilidade a ela e reconhecimento de suas competências, quando da exposição ao mercado internacional, aumentando as possibilidades de atuar internacionalmente. É importante ressaltar que a empresa 2 está situada dentro de um parque tecnológico da uma universidade, e a empresa 1 nasceu em uma incubadora junto a uma universidade. 
INTERAÇÃO UNIVERSIDADE-EMPRESA: UMA ANÁLISE DE EMPRESAS DA INDÚSTRIA DE SOFTWARE NO RIO GRANDE DO SUL

Daniel Pedro Puffal - Vilmar Antonio Gonçalves Tondolo - Dusan Schreiber - Vania Gisele Bessi

\subsubsection{Dificuldades na relação com universidades}

Finalmente foram identificados os principais empecilhos ou fatores que impedem ou dificultam a cooperação entre empresas de software e as universidades. Os pesquisados apontaram que os fatores que mais dificultam a interação são a baixa velocidade com que a universidade trata os assuntos relativos ao tema e o desalinhamento dos objetivos entre universidade e empresa. Outros fatores importantes são: a excessiva burocracia universitária e diferença de linguagem entre universidade e empresa. Além destes fatores, ainda é relatada pelos entrevistados a falta de recursos humanos para gerenciar o processo, a incerteza em relação ao resultado da interação e a falta de recursos da empresa para investir em tecnologia.

Ainda foram relatadas dificuldades em relação à propriedade intelectual e à falta de uma política governamental de apoio a interação com a universidade, mas, durante a discussão com os entrevistados, percebeu-se que talvez esta dificuldade esteja ligada a falta de conhecimento dos entrevistados referente às possibilidades e ofertas existentes.

\section{CONSIDERAÇÕES FINAIS}

As áreas de conhecimento em que há maior número de grupos de pesquisa com interação com empresas, ligadas às universidades e registradas no CNPq, são Engenharias e Ciências Agrárias, o que é compatível com o modelo de industrialização brasileiro. Porém, a área de Ciências da Computação, apesar de possuir menor número de grupos e menor percentual de IUE, ocupa o terceiro lugar. Também a localização geográfica indica que o número maior de grupos está localizado nos locais mais industrializados, entretanto, não é nestes locais que, percentualmente, há maior número de grupos com IUE. 
Como o presente artigo tinha o objetivo de analisar a interação entre universidade e pequenas e médias empresas de software instaladas no Rio Grande do Sul, o papel desta interação nos resultados das empresas e as características que distinguem estas interações com as das demais empresas brasileiras, observou-se que com relação ao desenvolvimento de produtos, nenhuma das empresas gaúchas de software entrevistadas possui estrutura específica destinada ao desenvolvimento e às pesquisa, estando essas atividades sob responsabilidade dos sócios das empresas que possuem formação específica na área. A constatação difere da encontrada entre as empresas do restante do Brasil, em que $67,1 \%$ das empresas pesquisadas declararam possuir departamento de P\&D.

Analisando as razões para interação com as universidades, constatou-se que as empresas de software gaúchas declararam que interagem com as universidades principalmente pela possibilidade de acesso aos recursos relativos à promoção e comercialização de seus produtos, além de acesso aos recursos financiados com recursos públicos. Estas razões diferem das do restante das empresas brasileiras, que indicaram que as principais razões para interação são a busca de recursos para a ampliação da capacidade de desenvolver tecnologia com menor investimento, menor prazo e menores riscos.

Com relação aos tipos de interação que as empresas têm com as universidades, as empresas de software gaúchas diferem das do restante do Brasil devido a maior informalidade das relações com a universidade, além de serem menos estruturadas são mais simples, menos intensas e de menor complexidade. As empresas gaúchas de software atribuem maior importância às atividades de capacitação e treinamento oferecidas pelas universidades, levando a concluir que as universidades são vistas como instituições cujo papel básico é a educação e não tanto como de apoio ao desenvolvimento tecnológico propriamente dito.

Destaca-se a baixa importância das relações com a universidade com o uso de informações sobre patentes pelos dois grupos de empresas. Isso pode ser atribuído às características históricas da estrutura brasileira de registro de propriedade intelectual, cujas empresas consideram a velocidade de trâmite inadequada se comparada com a que os negócios necessitam, além da conhecida morosidade do trâmite de processos em caso de 
uma disputa judicial de propriedade, optando assim a proteger sua propriedade intelectual por meio de sigilo.

Com relação aos resultados obtidos pelas empresas com interação com universidades, as empresas de software gaúchas também diferem das do restante do Brasil por estarem primeiramente preocupadas com a melhoria da imagem pública que a empresa pode adquirir, advinda da relação com a universidade, bem como da utilização de recursos disponibilizados pela universidade, do que com a produção de novos produtos ou processos.

Observou-se também a falta de expectativa, por parte das empresas gaúchas de software pesquisadas, de que a interação com as universidades possa reduzir os prazos ou custos para desenvolvimento de tecnologia, que ocorra o acesso aos resultados de pesquisas anteriores, ou ainda que haja um compartilhamento nos riscos com a universidade.

Finalmente, observou-se que, há espaço para um avanço nas relações entre as empresas de software e a universidade, que, apesar da proximidade física entre os agentes, as empresas entrevistadas ainda se caracterizam por um baixo nível de inovação de seus produtos se comparadas às demais empresas brasileiras. Porém, a existência de alguma penetração do setor no mercado internacional pode indicar que a interação necessária talvez seja mais no sentido de propiciar inovação nos processos das empresas do que no desenvolvimento direto de produtos para o mercado.

Como principais limitações do artigo, pode-se destacar o pequeno número de empresas do setor de software que participaram da pesquisa, bem como a restrita área de abrangência geográfica, o que, apesar de impossibilitar a generalização, proporciona uma melhor compreensão das relações universidade-empresa no setor em estudo.

Sugere-se que o estudo seja aprofundado, analisando as razões que levam as universidades a interagirem com as empresas de software, além dos resultados obtidos por elas, já que, a partir deste estudo, pode-se concluir que as empresas gaúchas de software pesquisadas pouco esperam das universidades no sentido de inovação em produtos e processos.

UNIVERSITY INDUSTRY LINKAGES: EVIDENCES FROM SOFTWARE INDUSTRY IN THE SOUTH OF BRAZIL. 


\section{ABSTRACT}

The software industry is characterized by innovation, technology-intensive, and its proximity to higher education institutions. Although embedded in a market economy where the main locus of innovation is a company, history has shown that, generally, companies cannot be trained by themselves; it requires the existence of a national innovation system that helps to develop this activity. In this context, the linkage among companies and universities plays a central role. Through analysis of a database on university-industry linkage in Brazil and multiple case studies, this article aims to observe the linkage among universities and small and medium-sized software companies, active in the international market, located in Rio Grande do Sul. Results indicate that the linkage among universities and software SMEs located at South of Brazil are less intense, more informal and less complexity, comparing them with the rest of Brazilian companies. Was also observed that there is opportunity for a breakthrough in relationships among companies and universities in order to increase the level of innovation of products and processes.

Key-words: Innovation. University industry linkages. software industry.

\section{REFERÊNCIAS}

ASHEIM, B.; GERTLER, M. Understanding regional innovation systems. In: FAGERBERG, J. MOWERY, D. NELSON, R. Editors. The Oxford Handbook of innovation. New York: Oxford University Press, 2007.

AXELROD, R. The evolution of cooperation. New York: Basic Books, 1984.

BARDIN, Laurence. Análise de conteúdo. Lisboa: Edições 70, 1977. 
INTERAÇÃO UNIVERSIDADE-EMPRESA: UMA ANÁLISE DE EMPRESAS DA INDÚSTRIA DE SOFTWARE NO RIO GRANDE DO SUL

Daniel Pedro Puffal - Vilmar Antonio Gonçalves Tondolo - Dusan Schreiber - Vania Gisele Bessi

BARNEY, J. B. Firm resources and sustained competitive advantage. Journal of Management, Vol. 17, pp. 99-120, 1991.

BELDERBOS, R.; CARREE, M.; LOKSHIN, B. Cooperative R\&D and firm performance. Research Policy, v. 33, pp. 1477-1492, 2004.

BONACCORSI, A.; PICCALUGA, A. A theoretical framework for the evaluation of universityindustry relationships. R\&D Management, v. 24, n. 3, p. 229-247, 1994.

CNPQ. Plano Tabular do Diretório de Grupos de Pesquisa do Brasil. Disponível em: <http://dgp.cnpq.br/planotabular/>. Acesso em: 20 jan. 2012.

COHEN, W.; NELSON, R.; WALSH, J. Links and impacts: the influence of public R\&D on industrial research. Management Science, v. 48, n. 1, pp. 1-23, 2002.

CORTEZIA, S. L. D. Internacionalização e Aprendizagem: um estudo sobre as micro e pequenas empresas da indústria de software do Estado do Rio Grande do Sul (Brasil). $188 \mathrm{f}$. Dissertação (Mestrado em Administração). UNISINOS, São Leopoldo, 2007.

COVIELLO, N. E.; MUNRO, H. Growing the Entrepreneurial Firm: Networking for International Market Development. European Journal of Marketing, v. 29, n. 7. p. 49-61. 1995.

DODGSON, M. Technological collaboration in Industry: Strategy, policy and internationalization in innovation. Ed. Routledge. 1993.

EISENHARDT, K. M.; GRAEBNER, M. E. Theory building from cases: opportunities and challenges. Academy of Management Journal, 50 (1), p. 25-32, 2007.

. Building Theories from Case Study Research. Academy of Management Review, v.14, n.4, p.532-550, 1989.

ETZKOWITZ, H. Innovation in Innovation: The triple Helix of University-Industry- Government Relations. Social Science Information, v.42, n.3, p. 293-337, 2003.

EVANS, D.; STARBUCH, E.; KIRESUK, T.; GEE, R. Center for interfacial engineering: an experiment in building industry-university partnerships. International Journal of Technology Management, v. 8, p. 622-651, 1993.

FERNANDES, A. C. et al. (2010) Academy industry links in Brazil: Evidence about channels and benefits for firms and researchers. Science and Public Policy, v. 37, N 7, p. 485-498, 2010.

FLEURY, A., e FLEURY, M. T. (Ed.). Internacionalização e os países emergentes. São Paulo: Atlas, 2007. 
INTERAÇÃO UNIVERSIDADE-EMPRESA: UMA ANÁLISE DE EMPRESAS DA INDÚSTRIA DE SOFTWARE NO RIO GRANDE DO SUL

Daniel Pedro Puffal - Vilmar Antonio Gonçalves Tondolo - Dusan Schreiber - Vania Gisele Bessi

FREEMAN, C. Technology Policy and Economic Performance: lessons from Japan. London/New York: Pinter Publishers. 1987.

GEISLER, E. Explaining the generation and performance of intersector technology cooperation: a survey of the literature. Technology Analysis \& Strategic Management, v. 13, $n^{\circ} 2$, p. 195-206, 2001.

. Industry-university technology cooperation: a theory of inter-organizational relationships. Technology Analysis \& Strategic Management; v. 7, º 2, p. 217-229, 1995.

KLEVORICK, A. K.; LEVIN, R.; NELSON, R. R.; WINTER, S. On the sources and significance of inter-industry differences in technological opportunities. Research Policy, v. 24, n. 2, p. 185205, March, 1995.

LUNDVALL, B-A. National Innovation Systems - Analytical concept and development tool. Industry and Innovation. V. 14, n. 1, p. 95-119, 2007.

. Sistemas nacionales de innovación y aprendizaje institucional. Comercio Exterior, agosto 1994.

MALERBA, F. Public policy and the development and growth of sectoral systems of innovation. Globelics Conference, Innovations Systems and Development, Beijing, 2004. Sectoral Systems of Innovation and Production, Research Policy, no 31, 2002.

MANSFIELD, E. \& LEE, J.Y., The Modern University: contributor to industrial innovation and recipient of industrial R\&D support. Research Policy v. $25 n^{\circ}$. 7, p. 1047-1058, 1996.

MAZZOLENI, R.; NELSON, R. The Roles of Research at Universities and Public Labs in Economic Catch-up. Research Policy, v. 36, n. 10, pp. 1512-1528, 2007.

MEYER-KRAMER, F e SCHMOCH, U. Science-based technologies: university-industry interactions in four fields. Research Policy, v. 27, n. 8, p. 835-85, dez, 1998.

MILLS, J. et al. Competing through competences. Cambridge: Cambridge University Press, 2002.

MOWERY D. C. e SAMPAT, B. N. Universities in national innovations systems. In: FAGERBERG,

J. MOWERY, D. NELSON, R. Editors. The Oxford Handbook of innovation. New York: Oxford University Press, 2007.

MYTELKA, L. Local systems of innovation in a globalized world economy. Industry and Innovation. n. 7, p. 15-32, 2000. 
INTERAÇÃO UNIVERSIDADE-EMPRESA: UMA ANÁLISE DE EMPRESAS DA INDÚSTRIA DE SOFTWARE NO RIO GRANDE DO SUL

Daniel Pedro Puffal - Vilmar Antonio Gonçalves Tondolo - Dusan Schreiber - Vania Gisele Bessi

NELSON, R. R. Sistemas Nacionais de Inovação: Retrospecto de um estudo. In: Nelson, R. R. As fontes do crescimento econômico. Campinas: Editora da Unicamp, 2006.

Press, 1993.

National Innovation Systems: a comparative analysis. Oxford: Oxford University

. Capitalism as an engine of progress. Research Policy. N. 19, p-193-214. 1990.

OECD, 2003. Science, Technology and Industry Scoreboard. Paris: OECD Publications.

2005. Oslo Manual: Guidelines for collecting and interpreting innovation data.

$3^{\text {th }}$ edition. France: OECD Publications.

PAVITT, K. Sectoral patterns of technical change: towards a taxonomy ande a theory.

Research Policy, v. 13, p. 343-373, 1984.

PENROSE, E. The theory of the growth of the firm. Oxford: Oxford University Press, 1959.

PROCHNICK, V., Esteves, L. A., \& Freitas, F. M. O grau de internacionalização das

firmas industriais brasileiras e suas características microeconômicas. In: De Negri, J. A., \&

Araújo, B. C. P. O. (Ed.). As empresas brasileiras e o comércio internacional. pp. 341-369.

Brasília: IPEA, 2006

RAPINI, M. S.; RIGHI, H. M. Metodologia e apresentação da base de dados do Censo 2004 do Diretório dos Grupos de Pesquisa do Conselho Nacional de Desenvolvimento Científico e Tecnológico (CNPQ). In: SUZIGAN, W.; ALBUQUERQUE, E. M. e CARIO, S. A. F. (Org). Em Busca da Inovação: Interação universidade-empresa no Brasil. Belo Horizonte: Autêntica Editora, 2011.

RIGHI, H. M. e RAPINI, M. S. Interação universidade-empresa no Brasil em 2002 e 2004: Uma aproximação a partir dos grupos de pesquisa do CNPq. Revista Economia, v. 8, n. 2, p. 248268, 2007.

ROSEMBERG, N. e NELSON, R. American university and technical advance in industry. Research Policy. N. 23, p 323-348. 1994.

SANTORO, M. D. Success breeds success: the linkage between relationship intensity and tangible outcomes in industry-university collaborative ventures. The Journal of High Technology Management Research, v. 11, nº. 2, p. 255-273, 2000.

SCHUMPETER, Joseph A. Theory of Economic Development. Harvard University Press, Cambridge. 1949.

STANKIEWICZ, R. Spin-off companies from universities. Science and Public Policy. n. 21, v. 2, p. 99-107, 1994.

R. eletr. estrat. neg., Florianópolis, v.5, n.2, p. 200-228, mai./ago. 2012 
INTERAÇÃO UNIVERSIDADE-EMPRESA: UMA ANÁLISE DE EMPRESAS DA INDÚSTRIA DE SOFTWARE NO RIO GRANDE DO SUL

Daniel Pedro Puffal - Vilmar Antonio Gonçalves Tondolo - Dusan Schreiber - Vania Gisele Bessi

SUZIGAN, W e ALBUQUERQUE, E. M. The underestimated role of universities for the Brazilian system of innovation. Revista de Economia Política. v. 31, no.1, p.03-30, Mar 2011.

TETHER, B. S. Who Co-operates for Innovation, and Why: An Empirical Analysis. Research Policy, v. 31, p. 947-967, 2002.

TÖDTLING, F. e TRIPPL M. One size fits all? Towards a differentiated regional innovation policy approach. Research Policy, n. 34, p. 1203-1219, 2005. 